\title{
Development of a Transgenic Flammulina velutipes Oral Vaccine for Hepatitis B
}

\section{Li-Hsin Huang' ${ }^{1}$, Hao-Yeh Lin², Ying-Tzu Lyu', Chiau-Ling Gung ${ }^{1}$ and Ching-Tsan Huang ${ }^{2 *}$}

'MycoMagic Biotechnology Co. Ltd., 8F-1, 12, Lane 270, Sec. 3, Beishen Road, New Taipei City, Taiwan

2Department of Biochemical Science and Technology, National Taiwan University, 1, Sec. 4, Roosevelt Road, Taipei, Taiwan

Received: 24 May 2018 Accepted: 31 January 2019

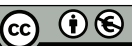

*Corresponding author:

Phone: +886233664454

Fax: +886223634796

E-mail: cthuang@ntu.edu.tw

ORCID IDs: 0000-0003-4593-9268

(Huang LH), 0000-0001-6093-1673 (Lin), 0000-0002-2030-0137 (Lyu), 0000-0002-9504-7615 (Gung), 0000-0003-1019-784X (Huang CT)

\section{SUMMARY}

Orally administered fungal vaccines show promise for the prevention of infectious diseases. Edible mushrooms are deemed appropriate hosts to produce oral vaccines due to their low production cost and low risk of gene contamination. However, their low expression level of antigens has limited the potential development of oral vaccines using mushrooms. The low expression level might result from impurity of the transgenic mycelia since dikaryotic mycelia are commonly used as transformation materials. In this study, stable transgenic hepatitis B virus surface antigen ( $\mathrm{HBsAg}$ ) in Flammulina velutipes transformants was obtained by Agrobacterium-mediated transformation, followed by fruiting and basidiospore mating. The formation of HBsAg was detected by western blot analysis. The expression levels of

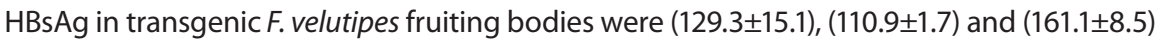
$\mathrm{ng} / \mathrm{g}$ total soluble protein. However, the values may be underestimated due to incomplete protein extraction. Two of the four pigs in the experimental group produced positive anti-HBsAg-specific lgG after being fed the HBsAg transgenic $F$. velutipes fruiting bodies for 20 weeks, while no anti-HBsAg antibody was detected in the control group. One of the positive pigs had HBsAg titres of 5.36 and $14.9 \mathrm{mlU} / \mathrm{mL}$ in weeks 10 and 14 , respectively, but expression faded thereafter. The other positive pig displayed HBsAg titres of 9.75, 17.86 and $39.87 \mathrm{mIU} / \mathrm{mL}$ in weeks 14,18 and 20, respectively. The successful immunogenicity in pigs fed transgenic $F$. velutipes fruiting bodies demonstrated the potential of using the fungus as an oral vaccine.

Key words: Agrobacterium-mediated transformation, Flammulina velutipes, hepatitis B, mating, oral vaccine

\section{INTRODUCTION}

Although vaccinations have saved millions of lives from infectious diseases, full implementation of global vaccination remains a challenge due to the relatively high costs of conventional vaccinations incurred by mass production, refrigeration and transportation, as well as by training and compensating personnel for their administration. To overcome these problems, many efforts have been devoted to the development of novel and cost-effective vaccination procedures and technologies. In addition to injected vaccines, oral administration of either raw materials or extracts from transgenic plants, spirulina or yeasts has been reported to stimulate systemic and mucosal immunity (1).

Oral vaccines are known to stimulate multiple types of immunity, including mucosal and humoral immunity (2). Plant-based oral vaccines were first proposed two decades ago (3). The use of transgenic fruits and vegetables for the expression of recombinant protein antigens as oral vaccines has become an attractive topic in plant molecular farming $(1,4)$. For example, many efforts have been made to develop hepatitis $B$ oral vaccines by expressing hepatitis B virus surface antigen ( $\mathrm{HBs} A \mathrm{~g})$ in transgenic plants such as tobacco, tomato, potato, banana, tomatillo and rice (5-12). In addition to plants, edible mushrooms are also appropriate hosts for the development of oral vaccines (13). Using transgenic mushrooms as oral vaccines has all the advantages of a plant-based system coupled with unique benefits, such as complete duplication, fast growth, scaled-up production under controlled 
conditions and less gene contamination. A previous study demonstrated the effectiveness of extracts of mycelia derived from edible mushrooms as adjuvants for intranasal influenza vaccines (14). Pérez-Martínez et al. (15) provided a comprehensive review on the use of Pleurotus as carrier of oral subunit vaccines. While mushroom-based oral vaccines seem promising, the low expression level of antigens and the instability of the transformants limit the development of mushroom molecular farming. Previously, we demonstrated that heterologous protein expression in the enoki mushroom Flammulina velutipes is notably enhanced by using $2 \mathrm{~A}$ peptide-mediated cleavage to co-express multiple copies of a single gene (16). Using this polycistronic expression strategy, enterovirus 71 virus-like particles were successfully produced in transgenic F. velutipes (17).

Mating and fruiting body formation are sexual stages in the life cycle of basidiomycete fungi and lead to karyogamy, meiosis and basidiospore formation. F. velutipes is a heterothallic basidiomycete fungus whose life cycle is characterized by haploid and diploid stages (18). F. velutipes has a tetrapolar mating type system that generates basidiospores with four possible mating types. After germination, the haploid spores develop into monokaryotic mycelia with $A x B x, A x B y, A y B x$ or AyBy mating types. Although single mating types have been known to produce fruiting bodies under severe stress, most fruiting requires the plasmogamy of two genetically different monokaryotic mycelia, AxBx/AyBy or AxBy/AyBx, to form the dikaryotic mycelia that consist of two nuclei in a cell and the structure of clamp connection (19). The generation of transgenic mushrooms usually involves Agrobacterium-mediated transformation using modified mycelial pellets, followed by the selection of transformants under appropriate pressures (17). Transformants are selected if the foreign gene is inserted into the chromosomal DNA in at least one nucleus of the mycelia. Consequently, the expression level and stability of heterologous genes are reduced if the transformants contain both transgenic and non-transgenic cells. Therefore, it is crucial to obtain transformants containing the transgene in all nuclei.

In this study, stable HBsAg transgenic F. velutipes transformants were obtained by Agrobacterium-mediated transformation, followed by fruiting and basidiospore mating. We demonstrated that specific immunogenicity was detected in pigs after feeding $\mathrm{HBs} A g$ transgenic $F$. velutipes fruiting bodies for a period of time.

\section{MATERIALS AND METHODS}

\section{Strains and media}

Flammulina velutipes BCRC 930110, a patented strain deposited at the Bioresource Collection and Research Centre (Hsinchu, Taiwan), was grown and maintained on complete yeast medium (CYM) agar or in broth containing $0.2 \%$ tryptone (BD Bioscience, Sparks, MD, USA), $0.2 \%$ yeast extract (Bio Basic, Amherst, NY, USA), 1 \% maltose (Sigma-Aldrich, Merck, St. Louis, MO, USA), and $2 \%$ glucose (Bio Basic) at $25^{\circ} \mathrm{C}$. Escherichia coli DH5a (Gibco BRL, Life Technologies, Grand
Island, NY, USA), which was used for DNA manipulation and plasmid conservation, was grown in Luria-Bertani (LB) medium (Sigma-Aldrich, Merck) at $37^{\circ} \mathrm{C}$. Agrobacterium tumefaciens strain LBA4404, kindly provided by Dr Yee-Yung Charng, Agricultural Biotechnology Research Centre, Academia Sinica (Taipei, Taiwan), was used for transformations and was grown in LB medium at $28^{\circ} \mathrm{C}$.

\section{Plasmid construction}

A promoter region of the Agaricus bisporus glyceraldehyde-3-phosphate dehydrogenase (Agpd) gene was amplified from the genome of $A$. bisporus by using primers Agpd-f (5'-TTAAGAGGTCCGCAAGTAGATTGA-3') and Agpd-r (5'-ATGTGTGTTGTTCGAATAGCGG-3'). The plasmid pGEM-Agpd was constructed by cloning the Agpd promoter into a pGEM-T Easy vector (Promega, Madison, WI, USA). The E. coli hygromycin B phosphotransferase (hpt) gene with cauliflower mosaic virus (CaMV) 35S terminator was amplified from pCAMBIA 1300 (Cambia, Canberra, Australia) using primers Spel-Hpt-f (5'-ACTAGTATGAAAAAGCCTGAACTCACC-3') and Pstl-CaMV 35 s terminator-r (5'-TTACACAATAATTCAACAGACGTCGG-3'). Then, the amplified fragment was cloned into a pGEM-Agpd by restriction enzymes Spel and Pstl. The backbone plasmid p0390-AH was constructed by introducing the hpt and CaMV 35 S terminator gene driven by Agpd promoter into the pCAMBIA0390 (p0390) vector (CAMBIA) by using restriction enzymes Ncol and Pstl. The F. velutipes gpd (Fgpd) promoter was amplified from the $F$. velutipes genome by using primers Ncol-Fgpd-f (5'-CCATGGGCATTACTTCGCTCTA-3') and Kpnl-Fgpd-r (5'-CGGTACCTTGTAGATGAGGAG-3'). The plasmid pGEM-Fgpd was constructed by cloning the Fgpd promoter into a pGEM-T Easy vector. The codon usage of hepatitis $B$ virus surface antigen $(H B s A g)$ derived from plasmid pRc/CMV-HBs(S) (Aldevron, Fargo, ND, USA) was modified according to the codon bias of $F$. velutipes, and an endoplasmic reticulum (ER) retention signal (HDEL) was fused to the $\mathrm{C}$ terminus of $\mathrm{HBsAg}$ protein to improve its expression. The gene of $H B s A g$ was amplified by primers Kpnl-HB-f (5'-GGTACCGATGGAGAACATCACAT-3') and BstEll-HB-r (5'-GGTCACCTTACAGTTCATCATGACTAGCAA-3') and cloned into a pGEM-Fgpd by restriction enzymes Kpnl and BstEll. The construct carrying the gene encoding $\mathrm{HBs} A g$ (accession number MG717400) driven by the F. velutipes gpd promoter was cut from pGEM-Fgpd by restriction enzymes $\mathrm{Ncol}$ and BstEll inserted into $\mathrm{p} 0390-\mathrm{AH}$, and the resulting plasmid was designated as p0390-AH-FmHB. A map of the plasmid constructs is shown in Fig. 1.

\section{Transformation procedure}

Agrobacterium-mediated transformation (AMT) was performed as described by Chen et al. (20) with minor modifications. The $\mathrm{p} 0390$ or p0390-AH or p0390-AH-FmHB constructs were introduced into $A$. tumefaciens by electroporation (ECM 630; BTX, San Diego, CA, USA). The A. tumefaciens strains harbouring the target plasmids were cultivated in LB medium 


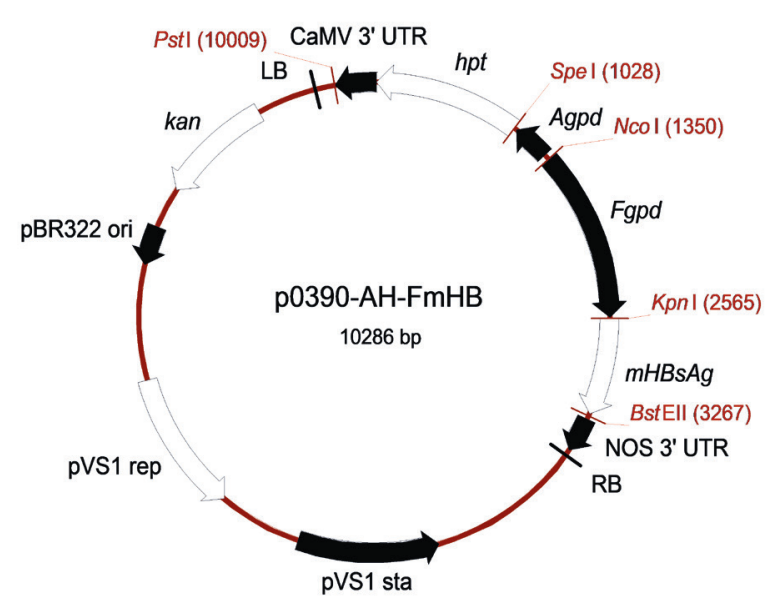

Fig. 1. A map of the plasmid construction: Agpd=the gpd promoter derived from Agaricus bisporus, Fgpd=the $g p d$ promoter derived from Flammulina velutipes, $m H B s A g=$ modified hepatitis $B$ virus surface

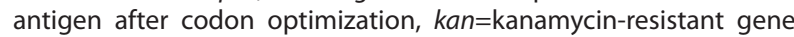
from Escherichia coli, hpt=hygromycin B phosphotransferase gene derived from $E$. coli, NOS 3' UTR=nopaline synthase $3^{\prime}$ UTR, CaMV $3^{\prime}$ UTR=cauliflower mosaic virus 355 terminator, pVS1 sta and pVS1 rep=stability and replication regions of vector $\mathrm{pVS1}$, respectively, pBR322 ori=origin of replication of vector pBR322, Pstl, Spel, Ncol, $K p n l$ and BstEll=restriction enzymes isolated from Providencia stuartii, Sphaerotilus sp., Nocardia corallina, Klebsiella pneumonia and Bacillus stearothermophilus, respectively, $\mathrm{LB}=$ left border, $\mathrm{RB}=$ right border. Numbers in brackets represent the position of the restriction sites

containing $50 \mu \mathrm{g} / \mathrm{mL}$ of kanamycin (MDBio Inc., Taipei, Taiwan) for $24 \mathrm{~h}$ at $28^{\circ} \mathrm{C}$, with shaking at $220 \mathrm{rpm}$, in an incubator (model S-300R; FIRSTEK, New Taipei City, Taiwan). The strains were then mixed with modified mycelial pellets (MMPs) of F. velutipes for $6 \mathrm{~h}$ at $23^{\circ} \mathrm{C}$ in an induction medium containing $11.8 \mathrm{mM}$ dipotassium phosphate, $10.7 \mathrm{mM}$ monopotassium phosphate, $2 \mathrm{mM}$ magnesium sulfate, $0.6 \mathrm{mM}$ calcium chloride, $9 \mu \mathrm{M}$ iron(II) sulfate, $43.7 \mathrm{mM}$ 2-(N-morpholino)-ethanesulfonic acid (MES; pH=5.3), $200 \mu \mathrm{M}$ acetosyringone (all from Sigma-Aldrich, Merck), $2.5 \mathrm{mM}$ sodium chloride, 3.8 $\mathrm{mM}$ ammonium sulfate, $0.18 \%$ glucose (all from Bio Basic), and $0.5 \%(\mathrm{~m} / \mathrm{V})$ glycerol (Riedel-de Haën; Honeywell, Morris, NJ, USA). After incubation, the MMPs were transferred to fresh induction medium agar plates and incubated at $23^{\circ} \mathrm{C}$ for 3-6 days. Then, the treated MMPs were washed with sterile water five times to remove bacteria, transferred to selective agar plates containing $30 \mu \mathrm{g} / \mathrm{mL}$ hygromycin B (Thermo Fisher Scientific, San Jose, CA, USA) and $200 \mathrm{mM}$ cefotaxime (MDBio Inc.) and incubated at $25^{\circ} \mathrm{C}$ for 2-3 weeks until hygromycin B-resistant mycelia of $F$. velutipes appeared.

\section{PCR analysis}

F. velutipes transformants grown on selective agar plates were subsequently screened via PCR analysis to confirm the integration of $H B s A g$ gene into the genomes. The $H B s A g$ gene fragment was amplified by 96-well thermal cycler (Veriti $^{T M}$ 9902; Applied Biosystems, Foster City, CA, USA) with primers HBsAg-f (5'-GAAGATCTATGGAGAACATCACATCC-3') and
HBsAg-r (5'-GTCGACGGGTCACCTTACAGTTCATCATGA-3'). Genomic DNA was extracted from four-week-old mycelia that were grown in CYM broth with $30 \mu \mathrm{g} / \mathrm{mL}$ hygromycin $B$, using the CTAB-mini DNA extraction method with minor modifications (21). The residual RNA in the isolated DNA was removed by treatment with DNase-free RNase A (GMbiolab Co., Ltd., Taipei, Taiwan).

\section{Mycelium staining}

Monokaryotic or dikaryotic mycelia on slides were fixed with $37 \%$ formaldehyde (Sigma-Aldrich, Merck) for $2 \mathrm{~min}$ at room temperature. Samples were then rinsed twice with sterile distilled water before incubation for $5 \mathrm{~min}$ at room temperature with $1 \mu \mathrm{g} / \mathrm{mL}$ 4',6-diamidino-2-phenylindole (DAPI; Sigma-Aldrich, Merck). The slides were observed by a fluorescence microscope (Eclipse E600; Nikon, Kanagawa, Japan) fitted with a Nikon UV-2A filter (330-380 nm excitation filter, $400 \mathrm{~nm}$ dichroic filter, and $420 \mathrm{~nm}$ barrier filter).

\section{Fruiting and mating of transformants}

The $F$. velutipes transformants were stabilized via mating. The fruiting body development procedure was described previously (22). Capped polypropylene bottles containing 70 $\%$ sawdust and $30 \%$ rice bran were purchased from Wanshen farm (Changhua, Taiwan), autoclaved for $1 \mathrm{~h}$, then inoculated with mycelial plugs and incubated at $23^{\circ} \mathrm{C}$ for approx. 3 to 4 weeks. After the vegetative mycelia had grown throughout the substrate, fruiting was induced by water addition, air exposure, and temperature change from 25 to $8^{\circ} \mathrm{C}$. The conditions were kept humid during the fruiting period by watering every other day with sterile distilled water. The basidiospores were collected in a sealed plate $(9-\mathrm{cm}$ Petri dishes) from the mature fruiting bodies and separated by resuspending them in sterile water. The suspension was then spread on CYM agar plates containing $30 \mu \mathrm{g} / \mathrm{mL}$ hygromycin B to form monokaryotic mycelia which were verified by DAPI staining. Mating was conducted by placing two mycelial plugs on the same CYM agar plate for 2 weeks at $23^{\circ} \mathrm{C}$. Successful mating was also confirmed by the formation of clamp connections and the presence of two nuclei in a cell. Three dikaryotic transformants were randomly picked for further analysis.

\section{Western blot analysis}

For western blot analysis of $\mathrm{HBsAg}$, the fruiting bodies of the $F$. velutipes transformants and of wild-type controls were collected and ground in liquid nitrogen with a mortar and pestle. A total of $40 \mathrm{mg}$ of sample powder was mixed on ice for $1 \mathrm{~h}$ with $0.5 \mathrm{~mL}$ of protein extraction buffer containing $50 \mathrm{mM}$ potassium phosphate ( $\mathrm{pH}=7.4$; Sigma-Aldrich, Merck), 0.1 \% Triton X-100 (J.T.Baker, Avantor, Radnor, PA, USA), 300 $\mathrm{mM}$ sodium chloride (Bio Basic), and $1 \mathrm{mM}$ phenylmethane sulfonyl fluoride (PMSF; Sigma-Aldrich, Merck). The total soluble protein (TSP) was obtained by centrifugation $(13000 \times g$ 
for 30 min at $4{ }^{\circ} \mathrm{C}$ ) using Thermo Scientific Sorvall ${ }^{\circledR}$ Legend Mach 1.6 R tabletop centrifuge (Thermo Fisher Scientific), boiled for 20 min with sample buffer containing $50 \mathrm{mM}$ Tris- $\mathrm{HCl}$ ( $\mathrm{pH}=7.4$; Bio-Rad, Hercules, CA, USA), $2 \%$ sodium dodecyl sulfate (SDS), $0.1 \%$ bromophenol blue, $10 \%$ glycerol, $400 \mathrm{mM}$ dithiothreitol (DTT) and $800 \mathrm{mM}$ 2-mercaptoethanol (all from Sigma-Aldrich, Merck), and separated by $12 \%$ sodium dodecyl sulfate polyacrylamide gel electrophoresis (SDSPAGE). The protein samples were transferred via a Trans-Blot ${ }^{\circledast}$ electrophoretic transfer cell (Bio-Rad) to a polyvinylidene difluoride (PVDF) membrane (Millipore, Bedford, MA, USA). Protein was detected using a mouse monoclonal anti-HBsAg antibody (ab9193; Abcam, Taipei, Taiwan) and a goat anti-mouse IgG antibody-conjugated alkaline phosphatase (ab97020; Abcam) in a reaction with 5-bromo-4-chloro-3-indolyl phosphate/nitro blue tetrazolium (BCIP/NBT) (PerkinElmer, Foster City, CA, USA) as described by the manufacturers.

\section{HBsAg quantification}

HBsAg was detected using the SURASE B-96 kit (General Biologicals Corporation, Taipei, Taiwan) according to the manufacturer's instructions. Plate wells coated with anti-HBsAg antibody were incubated with $50 \mu \mathrm{L}$ of TSP and anti-HBsAg antibody-conjugated peroxidase for $90 \mathrm{~min}$ at 37 ${ }^{\circ} \mathrm{C}$. Each assay was repeated in triplicate for each plate. After the incubation, the plate wells were washed six times with phosphate buffer (provided in the kit), then $100 \mu \mathrm{L}$ of the TMB One Component HRP microwell substrate (BioFX Laboratories Inc., Owings Mills, MD, USA) was added to each well, and the plates were incubated for $30 \mathrm{~min}$ at $37^{\circ} \mathrm{C}$. The reaction was stopped by the addition of $2 \mathrm{M}$ sulfuric acid (provided in the kit), and the absorbance at $450 \mathrm{~nm}$ of each well was measured using a 96-well plate reader (VERSAmax ${ }^{\mathrm{TM}}$; Molecular Devices, LLC, San Jose, CA, USA). Protein concentrations were determined using the Pierce ${ }^{\mathrm{TM}}$ BCA Protein Assay Kit (Pierce Biotechnology, Thermo Fisher Scientific, Rockford, IL, USA). The SURASE B-96 quantitative standard panel is included with each assay.

\section{Animal tests}

The animal tests were conducted at Animal Technology Laboratories, Agriculture Technology Research Institute (Chunan, Taiwan). Specific pathogen-free (SPF) pigs, four males and three females, three weeks old, $19 \mathrm{~kg}$ of average body mass, were provided by the Cheng-Yu Pig Farm (Hsinchu City, Taiwan). The fruiting bodies of transgenic F. velutipes with the highest $\mathrm{HBsAg}$ content were used for the animal tests. Four pigs, two males and two females, in the experimental group were each fed with $9 \mathrm{~g}$ dry transgenic $F$. velutipes fruiting body powder every 3 days for 6 consecutive weeks, followed by feeding once a week for another 14 consecutive weeks. Another three pigs fed with wild-type $F$. velutipes were used as the control group. The fruiting body powder was mixed with $2 \mathrm{~kg}$ fodder composed of corn, soybean and rice bran (Cheng-Yu Pig Farm), and left in the cage for 24 h or until all food was consumed. Regular fodder was used throughout the experiment. Starting from the sixth week, blood samples were collected periodically for antibody assay.

\section{Measurement of anti-HBsAg antibodies}

HBsAg antibodies were detected with Elecsys anti-HBs (Roche, Basel, Switzerland) electrochemiluminescence immunoassay according to the manufacturer's instructions using Modular E170 immunology analyzer (Roche). Anti-HBsAg antibodies in the sample, biotinylated HBsAg, and HBsAg labelled with a ruthenium complex reacted to form a sandwich complex. After the addition of streptavidin-coated microparticles, the complex bound to the solid phase via interaction of biotin and streptavidin. The reaction mixture was aspirated into the measuring cell where the microparticles were magnetically captured onto the surface of the electrode. Application of a voltage to the electrode induced chemiluminescent emission which was measured by Modular E170 immunology analyzer (Roche). Results were determined via a calibration curve which was instrument-specifically generated by 2-point calibration and a master curve provided via the reagent barcode, calculated in milli-international units per volume $(\mathrm{mlU} / \mathrm{mL})$, and reported as positive when the value was more than $10 \mathrm{mIU} / \mathrm{mL}$.

\section{Statistical analysis}

Least significant difference (LSD at $\mathrm{p}=0.05$ ) test was used to identify significant differences of the expression level among three transformants. Significant difference was defined at $\mathrm{p}<0.05$.

\section{RESULTS AND DISCUSSION}

\section{F. velutipes transformation}

A. tumefaciens containing p0390, p0390-AH or p0390-AH-FmHB was co-cultivated with $F$. velutipes MMPs in induction medium for 5-7 days and then transferred to selective agar plates containing $30 \mu \mathrm{g} / \mathrm{mL}$ hygromycin $\mathrm{B}$. The plasmids p0390 and p0390-AH were used as negative and positive control, respectively. As shown in Fig. 2, hygromycin B-resistant mycelia appeared approx. 2-4 weeks after the transfer to the selective agar plates; however, F. velutipes MMPs co-cultivated with $A$. tumefaciens carrying the empty vector p0390 failed to grow. To confirm the stability of the putative transformants carrying the plasmid p0390-AH-Fm$\mathrm{HB}$, they were subcultured three times on the agar plates under selection pressure to ensure that the hygromycin $B$ resistance gene and the $\mathrm{HBsAg}$ gene were stably expressed in $F$. velutipes. Even though the transformants can stably grow on the selective agar plates, their mycelia might be composed of both transgenic and non-transgenic cells. To resolve this problem, mushroom fruiting and basidiospore mating were conducted. 


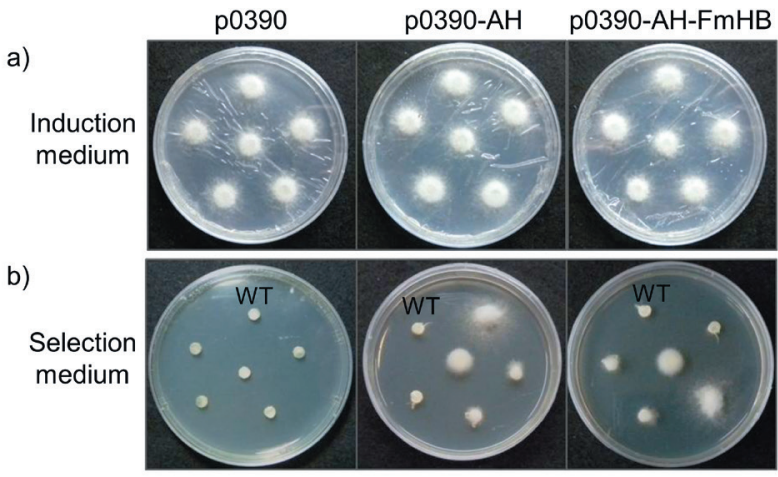

Fig. 2. The process of Agrobacterium tumefaciens-mediated transformation: a) Flammulina velutipes MMPs co-cultured with $A$. tumefaciens transformants (p0390, p0390-AH and p0390-AH-FmHB) on induction medium, b) co-cultured MMPs transferred onto selection medium with $30 \mu \mathrm{g} / \mathrm{mL}$ hygromycin B. WT=wild-type $F$. velutipes

\section{Transformant fruiting and mating}

Two putative transformants were selected as the parental strains for fruiting. The fruiting bodies of transformants 1 and 2 were cultivated and the basidiospores were collected. The basidiospores were separated by spreading them onto CYM agar plates containing $30 \mu \mathrm{g} / \mathrm{mL}$ hygromycin B to form monokaryotic mycelia. PCR analysis served to investigate the presence of the $H B s A g$ gene. Fig. 3 illustrates that $H B s A g$ gene was stably maintained in the parental (Fig. 3a) and the filial generation (Fig. 3b) of dikaryotic transformants, and in the monokaryotic mycelium transformants derived from parental generation (Fig. $3 \mathrm{c})$. The successful mating was checked by the clamp connection and by compatible growth of two monokaryotic mycelial plugs. No clamp connections were observed in the monokaryotic mycelia (Fig. 4a). Mating was conducted by placing two monokaryotic mycelial plugs on the same CYM agar plate containing $30 \mu \mathrm{g} / \mathrm{mL}$ hygromycin B and incubating at $23^{\circ} \mathrm{C}$ for 3 weeks. The successful mating was

a)

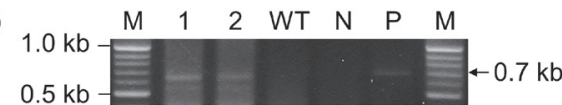

b)
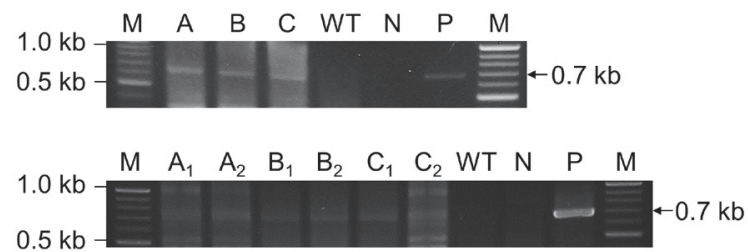

Fig. 3. PCR analysis of putative transformants: a) the parental generation of HBsAg Flammulina velutipes dikaryotic transformants, b) the filial generation of HBsAg F. velutipes dikaryotic transformants, and c) the monokaryotic mycelium transformants derived from parental generation. Lanes 1 and 2 =the parental generation of HBsAg F. velutipes dikaryotic transformants, lanes $A, B$ and $C=$ the filial generation of $\mathrm{HBsAg} F$. velutipes dikaryotic transformants, lanes $A_{1}, A_{2}, B_{1}, B_{2}, C_{1}$ and $C_{2}=$ the mating type of monokaryotic transformants of $A, B$ and $C$, lane $\mathrm{WT}=$ wild-type $F$. velutipes, lane $\mathrm{P}$ =positive control using plasmid p0390-AH-FmHB, lane $\mathrm{N}=$ negative control, and lane $\mathrm{M}=$ marker a)
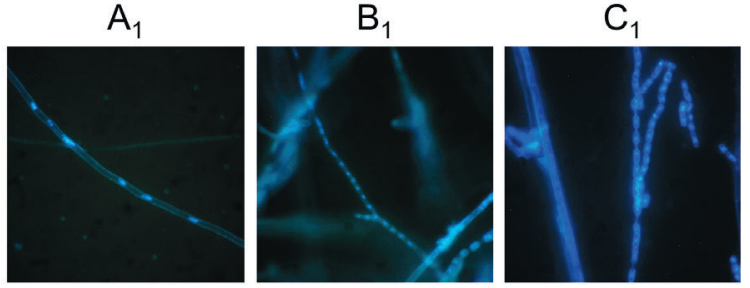

$\mathrm{A}_{2}$

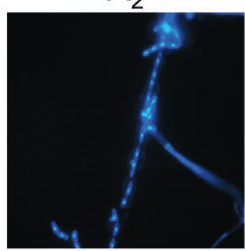

$\mathrm{B}_{2}$

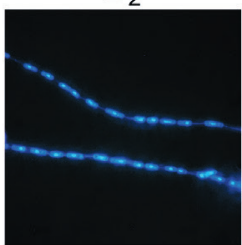

$\mathrm{C}_{2}$

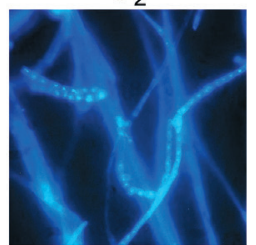

b)

A

B
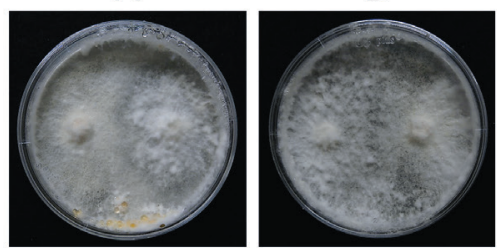

C

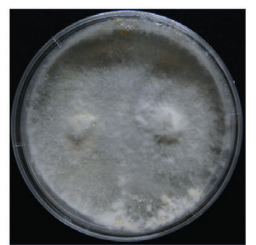

c)
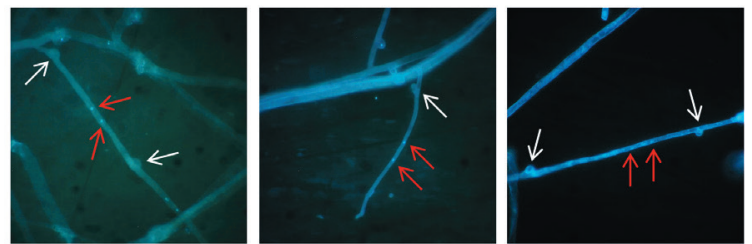

Fig. 4. The mating process of Flammulina velutipes transformants: a) microphotographs of monokaryotic mycelia of transformants stained by DAPI, b) morphology of the filial generation of $\mathrm{HBsAg} F$. velutipes transformants after mating, and c) microphotographs of filial generation of $\mathrm{HBsAg} F$. velutipes transformants stained by DAPI. Lanes $\mathrm{A}, \mathrm{B}$ and $\mathrm{C}=$ the filial generation of $\mathrm{HBsAg} F$. velutipes dikaryotic transformants, lanes $A_{1}, A_{2}, B_{1}, B_{2}, C_{1}$ and $C_{2}=$ the mating type of monokaryotic transformants of $A, B$ and $C$, white arrow=clamp connection, red arrow $=$ nucleus

confirmed by compatible growth between two monokaryotic mycelial plugs (Fig. 4b) and clamp connections (Fig. 4c). These results show that both nuclei in each pure cell of the filial dikaryotic mycelia contained the target gene. The dikaryotic mycelia containing the $H B s A g$ gene remained stable after repeated subculture (data not shown). The fruiting bodies of the stable dikaryotic mycelia were cultivated for further $\mathrm{HBsAg}$ analysis and for the animal tests.

\section{HBsAg quantification and qualification via ELISA and western blot analysis}

The western blot analysis of three HBsAg transgenic F. velutipes fruiting bodies is shown in Fig. 5 . Immunoblotting with the monoclonal anti-HBsAg antibody detected a band at 25.4 $\mathrm{kDa}$ in the positive control (without the endoplasmic reticulum (ER) retention sequence) and a band at $26.1 \mathrm{kDa}$ in the transformants, while no signal was observed in wild-type $F$. velutipes. The HBsAg expression level in the fruiting bodies of these three transformants was determined by ELISA (Fig. 6). 
a)

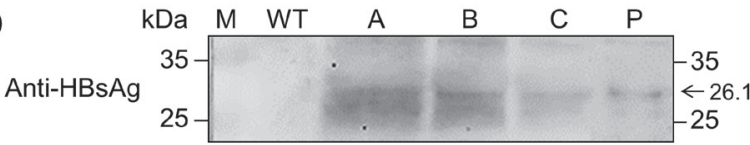

b)

Anti-GPD

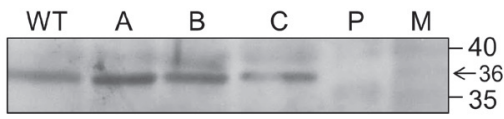

Fig. 5. Expression of HBsAg in Flammulina velutipes transformants: a) the western blot analysis of three $\mathrm{HBsAg}$ transgenic $F$. velutipes fruiting body immunoblotting with the monoclonal anti-HBsAg antibody, b) immunoblotting with the monoclonal anti-GPD antibody $\mathrm{GPD}=$ glyceraldehyde-3-phosphate dehydrogenase. Lane WT=wild type, lanes A, B and C=the filial generation of $\mathrm{HBsAg}$ F. velutipes transformants, lane $\mathrm{P}=$ positive control, a commercial HBsAg (226 amino acids, $25.4 \mathrm{kDa}$ ); the HBsAg expressed in transformants is 232 amino acids, $26.1 \mathrm{kDa}$, and lane $\mathrm{M}=$ marker

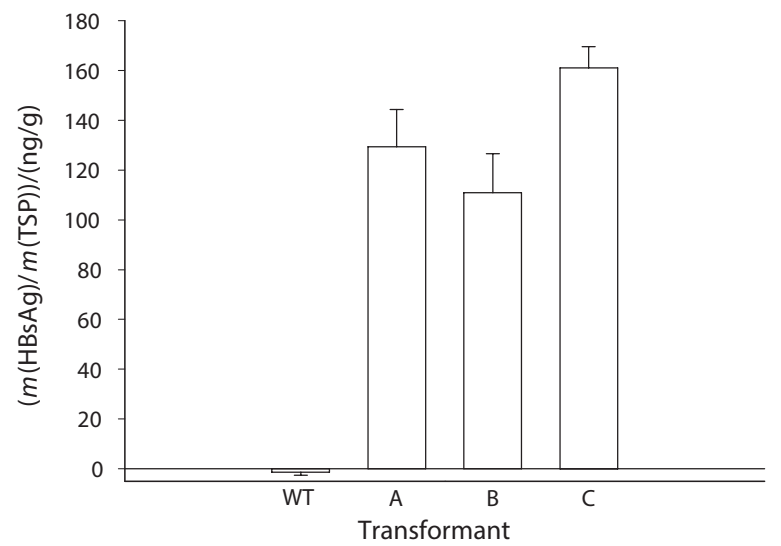

Fig. 6. HBsAg quantification of fruiting bodies for wild-type and transgenic Flammulina velutipes. WT=wild type, $A, B$ and $C=$ the filial generation of $\mathrm{HBsAg} F$. velutipes transformants, $\mathrm{TSP}=$ total soluble protein The HBsAg assays were repeated three times. The error bars indicate the standard deviation. Expression level among transformants $A$, $B$ and $C$ is not significantly different $(p>0.05)$

The HBsAg expression level of transformants $A, B$ and $C$ was (129.3 \pm 15.1$),(110.9 \pm 1.7)$ and $(161.1 \pm 8.5) \mathrm{ng} / \mathrm{g}$ TSP, respectively. Transformant $C$ was further used for the large-scale production of fruiting bodies for animal tests. The results indicate that stable HBsAg transgenic F. velutipes fruiting bodies were generated using monokaryotic mycelium selection and the basidiospore mating.
Previous research has expressed HBsAg in edible plant tissues, including lettuce leaves (23), cherry tomatillos and tomatoes (7), at less than $0.000001 \%$ of fresh mass, while in potato tubers it was up to $0.002 \%$ of fresh mass (5). The low antigen expression level might be attributed to molecular biology mechanisms such as codon bias, improper mRNA splicing and post-translational modification, or to impure transgenic mycelia. In this study, the codons of HBsAg were optimized by checking the CDNA sequences via reversed transcription PCR, thus eliminating the potential for improper mRNA splicing. Since the stability of HBsAg virus-like particles relies on disulphide bond formation in the ER (24), an ER retention signal, HDEL, was constructed to ensure the retention of HBsAg in the ER and increase the likelihood of forming the correct structure. In addition, pure $\mathrm{HBsAg}$ transgenic $F$. velutipes mycelia were obtained via a mating scheme. The low expression level may be a result of incomplete protein extraction and weak antibody recognition since the antigenic domain presented on hepatitis $B$ virus envelope is a conformational rather than a linear epitope (25). The development of more efficient $\mathrm{HBsAg}$ extraction and detection methods from transgenic $F$. velutipes fruiting bodies is required.

\section{Oral immunogenicity of HBsAg was stimulated by transgenic $F$. velutipes}

The immune response to the $\mathrm{HBsAg}$ transgenic F. velutipes fruiting bodies in pigs is shown in Table 1. No anti-HBsAg antibody was detected in the control group throughout the experiment. The blood sample collected from male pig 15903 in the experimental group displayed an antibody response value of $5.36 \mathrm{mIU} / \mathrm{mL}$ in week 10, which is below the positive response value (10 mlU/mL). The HBsAg-specific lgG level in this pig reached a maximum of $14.9 \mathrm{mIU} / \mathrm{mL}$ in week 14 and then decreased thereafter. Female pig 17215 had HBsAg levels of 9.75, 17.86 and $39.87 \mathrm{mlU} / \mathrm{mL}$ in weeks 14,18 and 20, respectively. The other two pigs in the experimental group did not produce detectable antibodies. These results provide solid evidence that a primary anti-HBsAg immune response can be stimulated in pigs by oral administration of transgenic $F$. velutipes fruiting bodies.

Table 1. Serum anti-HBsAg antibodies detected in pig

\begin{tabular}{|c|c|c|c|c|c|c|c|}
\hline \multirow{3}{*}{ Group } & \multirow{3}{*}{$\begin{array}{l}\text { Pig code } \\
\text { (gender) }\end{array}$} & \multicolumn{6}{|c|}{$t /$ week } \\
\hline & & 6 & 8 & 10 & 14 & 18 & 20 \\
\hline & & \multicolumn{6}{|c|}{$N($ antibody)/(mlU/mL) } \\
\hline \multirow{3}{*}{ Control } & $16113(\mathrm{~F})$ & 2.00 & 2.00 & 2.00 & 2.00 & 2.00 & 2.00 \\
\hline & $17202(\mathrm{M})$ & 2.00 & 2.00 & 2.00 & 2.00 & 2.00 & 2.00 \\
\hline & $17204(\mathrm{M})$ & 2.00 & 2.00 & 2.00 & 2.00 & 2.00 & 2.00 \\
\hline \multirow{4}{*}{ Experiment } & $16114(F)$ & 2.00 & 2.00 & 2.00 & 2.00 & 2.00 & 2.00 \\
\hline & $17215(F)$ & 2.00 & 2.00 & 2.00 & 9.75 & 17.86 & 39.87 \\
\hline & 15903 (M) & 2.00 & 2.00 & 5.36 & 14.90 & 2.00 & 2.00 \\
\hline & $17308(\mathrm{M})$ & 2.00 & 2.00 & 2.00 & 2.00 & 2.00 & 2.00 \\
\hline
\end{tabular}

$\mathrm{mIU}=$ milli-international units, $\mathrm{F}=$ female, $\mathrm{M}=$ male

Due to severe weather conditions no blood was collected in the 12th and 16th week 
Several studies have suggested that increased immunogenicity of HBsAg using oral administration via transgenic potatoes and tomatoes usually requires an adjuvant, such as cholera toxin (CT). Richter et al. (5) reported that feeding mice with $5 \mu \mathrm{g}$ HBsAg expressed in potato tuber and $10 \mu \mathrm{g}$ CT prompted a serum response peaking at approx. $70 \mathrm{mIU} / \mathrm{mL}$. In the absence of adjuvant, primary immunization with $40 \mu \mathrm{g}$ oral doses of potato-expressed antigen did not induce a response (6), but otherwise with $60 \mu \mathrm{g}$ oral doses antigen prompted an antibody response up to $170 \mathrm{mlU} / \mathrm{mL}$ (26). In this study, a positive response was obtained by feeding transgenic $F$. velutipes fruiting bodies without $\mathrm{CT}$, indicating that the mushroom polysaccharides might serve the role of an adjuvant. This observation is consistent with the report of Ichinohe et al. (14), who demonstrated the effectiveness of mycelial extracts derived from edible mushrooms as adjuvants for an intranasal influenza vaccine.

\section{CONCLUSION}

In this study, we obtained stable HBsAg transgenic F. velutipes dikaryotic mycelia via a mating scheme and confirmed that the production of anti-HBsAg antibodies was stimulated in pigs by feeding them $F$. velutipes fruiting bodies, demonstrating the potential of the application of $F$. velutipes in oral vaccines. This is the first report to show that the immunogenicity in pigs can be achieved by feeding them transgenic HBsAg mushroom.

\section{ACKNOWLEDGEMENTS}

The assistance of Dr Szu-Ming Chen (New Taipei City, Taiwan) in measuring the anti-HBsAg antibodies is greatly appreciated.

\section{FUNDING}

No external funding was received.

\section{CONFLICT OF INTEREST}

LHH is a current employee, and YTL and CLG are former employees of MycoMagic Biotechnology Co., Ltd. HYL is a former graduate student advised by CTH at National Taiwan University. CTH is bound with a Technology Transfer Agreement between MycoMagic and National Taiwan University (09T-101203-1N-A). The authors have declared that there are no other competing interests.

\section{ETHICS APPROVAL}

All animal tests were conducted by the Animal Technology Laboratories, Agriculture Technology Research Institute (Contract No.: BL-11-T020801-P) and were in compliance with Laboratory Animal Welfare and Ethics guidelines as issued by the Council of Agriculture, Taiwan. The protocol was approved by the Committee on Laboratory Animal Management of Agriculture Technology Research Institute, Taiwan.

\section{REFERENCES}

1. Daniell H, Streatfield SJ, Wycoff K. Medical molecular farming: production of antibodies, biopharmaceuticals and edible vaccines in plants. Trends Plant Sci. 2001;6(5):219-26. https://doi.org/10.1016/S1360-1385(01)01922-7

2. Pniewski T. Is an oral plant-based vaccine against hepatitis B virus possible? Curr Pharm Biotechnol. 2012;13(15):2692704.

https://doi.org/10.2174/138920112804724837

3. Arntzen CJ. High-tech herbal medicine: plant-based vaccines. Nat Biotechnol. 1997;15(3):221-2.

https://doi.org/10.1038/nbt0397-221

4. Joung YH, Park SH, Moon KB, Jeon JH, Cho HS, Kim HS. The last ten years of advancements in plant-derived recombinant vaccines against hepatitis B. Int J Mol Sci. 2016;17(10): Article no. 1715.

https://doi.org/10.3390/ijms17101715

5. Richter LJ, Thanavala Y, Arntzen CJ, Mason HS. Production of hepatitis $B$ surface antigen in transgenic plants for oral immunization. Nat Biotechnol. 2000;18(11):1167-71. https://doi.org/10.1038/81153

6. Kong Q, Richter L, Yang YF, Arntzen CJ, Mason HS, Thanavala Y. Oral immunization with hepatitis $B$ surface antigen expressed in transgenic plants. Proc Natl Acad Sci USA. 2001;98(20):11539-44.

https://doi.org/10.1073/pnas.191617598

7. GaoY, MaY, Li M, Cheng T, Li SW, Zhang J, et al. Oral immunization of animals with transgenic cherry tomatillo expressing HBsAg. World J Gastroenterol. 2003;9(5):996-1002.

https://doi.org/10.3748/wjg.v9.i5.996

8. Huang Z, Mason HS. Conformational analysis of hepatitis B surface antigen fusions in an Agrobacterium-mediated transient expression system. Plant Biotechnol J. 2004;2(3):241-9.

https://doi.org/10.1111/j.1467-7652.2004.00068.x

9. Kumar GBS, Ganapathi TR, Revathi CJ, Srinivas L, Bapat VA. Expression of hepatitis $B$ surface antigen in transgenic banana plants. Planta. 2005;222(3):484-93.

https://doi.org/10.1007/s00425-005-1556-y

10. Gleba Y, Klimyuk V, Marillonnet S. Viral vectors for the expression of proteins in plants. Curr Opin Biotechnol. 2007;18(2):134-41.

https://doi.org/10.1016/j.copbio.2007.03.002

11. Mett V, Farrance CE, Green BJ, Yusibov V. Plants as biofactories. Biologicals. 2008;36(6):354-8.

https://doi.org/10.1016/j.biologicals.2008.09.001

12. Tiwari S, Verma PC, Singh PK, Tuli R. Plants as bioreactors for the production of vaccine antigens. Biotechnol Adv. 2009;27(4):449-67.

https://doi.org/10.1016/j.biotechadv.2009.03.006 
13. Kilaru S, Collins CM, Hartley AJ, Bailey AM, Foster GD. Establishing molecular tools for genetic manipulation of the pleuromutilin-producing fungus Clitopilus passeckerianus. Appl Environ Microb. 2009;75(22):7196-204. https://doi.org/10.1128/AEM.01151-09

14. Ichinohe T, Ainai A, Nakamura T, Akiyama Y, Maeyama J, Odagiri $T$, et al. Induction of cross-protective immunity against influenza A virus $\mathrm{H} 5 \mathrm{~N} 1$ by an intranasal vaccine with extracts of mushroom mycelia. J Med Virol. 2010;82(1):128-37. https://doi.org/10.1002/jmv.21670

15. Pérez-Martínez AS, Acevedo-Padilla SA, Bibbins-Martinez M, Galván-Alonso J, Rosales-Mendoza S. A perspective on the use of Pleurotus for the development of convenient fungi-made oral subunit vaccines. Vaccine. 2015;33(1):25-33. https://doi.org/10.1016/j.vaccine.2014.10.059

16. Lin YJ, Huang LH, Huang CT. Enhancement of heterologous gene expression in Flammulina velutipes using polycistronic vectors containing a viral $2 \mathrm{~A}$ cleavage sequence. PLoS One. 2013;8(3):e59099. https://doi.org/10.1371/journal.pone.0059099

17. Lin YJ, Liu WT, Stark H, Huang CT. Expression of enterovirus 71 virus-like particles in transgenic enoki (Flammulina velutipes). Appl Microbiol Biotechnol. 2015;99(16):6765-74. https://doi.org/10.1007/s00253-015-6588-z

18. Petersen RH, Hughes KW, Redhead SA, Psurtseva N, Methven AS. Mating systems in the Xerulaceae (Agaricales, Basidiomycotina): Flammulina. Mycoscience. 1999;40(5):411-26. https://doi.org/10.1007/BF02464396

19. van Peer AF, Park SY, Shin PG, Jang KY, Yoo YB, Park YJ, et al. Comparative genomics of the mating-type loci of the mushroom Flammulina velutipes reveals widespread synteny and recent inversions. PLoS One. 2011;6(7):e22249. https://doi.org/10.1371/journal.pone.0022249

20. Chen X, Stone M, Schlagnhaufer C, Romaine CP. A fruiting body tissue method for efficient Agrobacterium-mediated transformation of Agaricus bisporus. Appl Environ Microb. 2000;66(10):4510-3.

https://doi.org/10.1128/AEM.66.10.4510-4513.2000

21. Kuo CY, Chou SY, Huang CT. Cloning of glyceraldehyde-3-phosphate dehydrogenase gene and use of the gpd promoter for transformation in Flammulina velutipes. Appl Microbiol Biotechnol. 2004;65(5):593-9.

https://doi.org/10.1007/s00253-004-1635-1

22. Kuo CY, Chou SY, Hseu RS, Huang CT. Heterologous expression of EGFP in enoki mushroom Flammulina velutipes. Bot Stud. 2010;51:303-9.

23. Kapusta J, Modelska A, Figlerowicz M, Pniewski T, Letellier $\mathrm{M}$, Lisowa $\mathrm{O}$, et al. A plant-derived edible vaccine against hepatitis B virus. FASEB J. 1999;13(13):1796-9. https://doi.org/10.1096/fasebj.13.13.1796

24. Huovila AP, Eder AM, Fuller SD. Hepatitis B surface antigen assembles in a post-ER, pre-Golgi compartment. J Cell Biol. 1992;118(6):1305-20.

https://doi.org/10.1083/jcb.118.6.1305

25. Le Bouvier GL. Subtypes of hepatitis B antigen: clinical relevance? Ann Intern Med. 1973;79(6):894-6.

https://doi.org/10.7326/0003-4819-79-6-894

26. Rukavtsova EB, Rudenko NV, Puchko EN, Zakharchenko NS, Buryanov YI. Study of the immunogenicity of hepatitis $B$ surface antigen synthesized in transgenic potato plants with increased biosafety. J Biotechnol. 2015;203:84-8. https://doi.org/10.1016/j.jbiotec.2015.03.019 